\title{
The Spatial Symmetry Axis of Earthquake Hazard in China
}

\author{
Junping Yan \\ College of Tourism and Environment Science, Shaanxi Normal University, Xi'an, Shaanxi, 710062, China \\ E-mail: yanjp@snnu.edu.cn \\ Shuangshuang Li, Jing Bai, Xinyan Liu \\ College of Tourism and Environment Science, Shaanxi Normal University, Xi'an, Shaanxi, 710062, China
}

\begin{abstract}
Natural disaster risk assessment is one of the hot fields of the study of natural disaster. It is of theoretical and practical significance to accurately identify the trend of natural disasters. Not all the natural disaster has the quality of spatial symmetry, only some disasters during some certain periods (active period) and some certain regions share this quality, which requires substantial research work to distinguish. This paper takes the spatial symmetry axis of earthquake hazard in china as the key of assessing natural disaster risk to emphatically analyze the rationality of the existence of the spatial symmetry axis of the regions of western china, Yunnan province and Taiwan and find out the dynamics basis of spatial symmetry axis combing with those presentational features, so as to provide some theoretical thinking and methods for reference to identify the time, spatial trends of the earthquake disaster occurrence. The results show that the earthquakes have the quality of spatial symmetry in certain regions and the symmetry has the basis of dynamics, which can work as the basis of the regional earthquake trend. And further improvements are needed to judge the earthquake trend to combine the spatial imagery, dynamics basis and physical mechanism..
\end{abstract}

Keywords: Earthquake; The spatial symmetry; Dynamics; Physical basis

\section{中国地震空间对称轴探讨 \\ 延军平 李双双 白晶 刘新颜 \\ 陕西师范大学/旅游与环境学院, 陕西西安, 710062}

\begin{abstract}
摘要: 在灾害的综合研究中, 自然灾害风险评价是灾害研究的一个热点领域, 对自然灾害进行准确的趋势 判断, 其理论和现实意义重大。不是所有灾害均具有时空对称性, 只有部分灾害在部分时期 (活跃期) 和 部分空间具有一定的对称性。这正需要我们进行大量的研究甄别。本文以中国地震空间对称轴为切入点, 着重探讨了中国西部地震空间对称轴、云南地震空间对称轴、台湾地震空间对称轴等存在的合理性, 结合 表象特征寻找空间对称轴的动力学基础, 从而为判断地震灾害未来发生的时间、空间趋势提供一些理论思 考和方法借鉴。结果表明, 地震在部分区域具有空间对称性特征, 其对称性有一定的动力学基础, 并且可 以作为区域地震未来趋势判断的依据。如何通过地震对称性的空间表象、动力学基础以及其物理机理有机 结合来判断地震趋势仍需进一步探索。
\end{abstract}

关键词: 地震; 空间对称; 动力学; 物理基础

\section{1. 问题的提出}

在灾害的综合研究中, 自然灾害风险评价是灾 害研究的一个热点领域, 其研究的关键是准确判断 重大自然灾害的发生趋势, 即研究未来 2 20 年内发 生重大自然灾害的可能年份和趋势。针对目前灾害 研究单一手段多, 综合方法少; 害的风险多, 灾的 风险少; 历史评价多, 趋势判断少; 学术研究多, 减灾价值少的现象, 如果能够提取出灾害的对称性
结构, 自此由表及里地逼近灾害发生的物理机理, 逐步提高重大自然灾害的趋势判断水平, 这可能是 地理学对灾害研究的重要贡献。

通常所说的对称性多指几何空间配置上的对称 性, 如数学中的点对称、轴对称等等, 是被观察对 象相对于某一事物经过空间位置转换后和变化前相 似的性质。时间对称性是指某些地理现象的产生和 变化存在一定的时间周期或多重时间周期叠加组 合, 在周期的任意对应时刻都存在相同或相似的性 
状或状态。对称性体现的就是一种万变中的不变。 值得一提的是, 笔者认为只有部分灾害在部分时期 （活跃期）和部分空间具有一定的对称性, 并不是 所有的灾害都具有时空对称性。这就需要通过深入 研究, 对不同案例进行甄别, 寻找对称性规律 ${ }^{1}$ 。

物理原理、物理基础与物理机理是三个既有联 系又存在不同的概念。物理原理是宏观机理, 物理 机理是具体的动力学过程, 物理基础则是介于二者 之间的中间状态, 物理基础是认识物理机理的基 础。认识地震时空对称结构的物理基础, 是探索地 震对称性物理机理的前提, 空间等间距现象则是形 成对称轴的基础。物理基础是强震灾害趋势判断的 理论前提, 物理机理是强震灾害趋势预测的理论基 础（图 1)。我们的研究在此定位为物理基础。

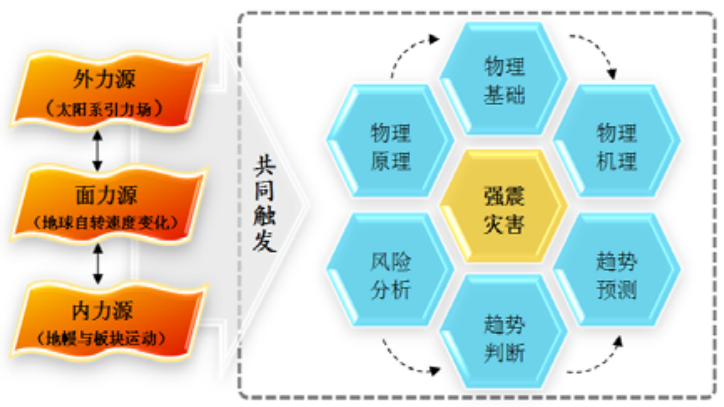

图 1 强震灾害分析阶段与体系

强震灾害风险分析、风险评价、趋势判断的关 系。强震灾害风险分析是指充分利用人类对各种致 灾因子（强震）、承灾体和社会系统的研究成果, 对一定区域、工程项目等可能遭受强震灾害的程度 进行可能性意义下的量化分析, 并对采取减灾措施 后的可能效果也进行分析。强震灾害风险评价 (Risk Assessment of Major Earthquake Disaster) 则是指通过 风险分析的手段或观察外表法, 对尚未发生的强震 灾害之致灾因子强度、受灾程度, 进行评定和估 计。评定是根据致灾因子强度和承灾体脆弱性推断 出受灾程度。估计是不确定意义下的估计。强震灾 害风险分析是自然灾害学和风险科学的集成体, 重 点在科学理论方面。强震灾害风险评价是风险分析 技术在自然灾害学中的应用, 重点在具体的模型方 面, 属于技术层面 ${ }^{2}$ 。

强震趋势判断与强震风险分析一样, 均属于理 论方面研究, 但是两者的侧重点有所不同。强震趋 势判断侧重于未来强震什么时间发生、地点在哪里 和强度如何; 强震风险分析侧重于怎么办, 即未来 强震发生后, 承灾体的脆弱性如何, 承载力如何, 能否应对; 如果不能, 如何改善、提高等问题。如 果强震趋势判断能取得关键性的突破, 其对强震风 险分析将有非常大促进。
马宗晋认为: “地震分区是探索区域地震成因 和区域地球动力学的一种途径”。地震分区的原则 之一就是对每一地震分区给予适当的动力学解释。 笔者曾通过可公度法计算, 于 2008 年在《陕西师范 大学学报》 (哲学社会科学版) 发表“关于地震预测 体系构建的可能性及验证”论文 ${ }^{3}$, 认为 2010 年中国 西部 (即川滇地区) 将发生 $\mathrm{Ms} \geqslant 7.2$ 地震, 结果是 2010 年 4 月 14 日青海玉树发生 7.1 地震, 其震级误 差在 0.1 级内, 只是位置略偏西北。本文在已有的 地震分区研究基础上, 着重探讨了中国西部地震空 间对称轴、云南地震空间对称轴、台湾地震空间对 称轴等存在的合理性, 即结合表象特征寻找空间对 称轴的动力学基础, 从而为判断地震灾害未来发生 的时间、空间趋势提供一些理论上的思考和方法上 的借鉴。

\section{2. 中国西部地震空间对称轴}

\section{1. 中国西部地震空间特征}

在大陆地质结构中, 中国西部及附近地区是一 个特殊的构造区域, 是一个相对完整的较大的构造 系。中国西部是印度板块运动的最大受力区, 是全 球板内地震最活跃的地区, 也是板内地震灾害的最 大受害区。在中国西部地区, 在地形结构上存在一 定的对称性分布, 大致以新疆拜城一青海德令哈一 四川广元三点连线为界, 可把中国西部划分为东 北、西南两个次一级的地震空间活动单元, 这条线 就是西北一东南地震空间对称轴, 简称为西北对称 轴 (图 2)。

该对称轴位于地块与活动带的交界处, 包括塔 里木块体与天山山地之间、柴达木块体与祁连山之

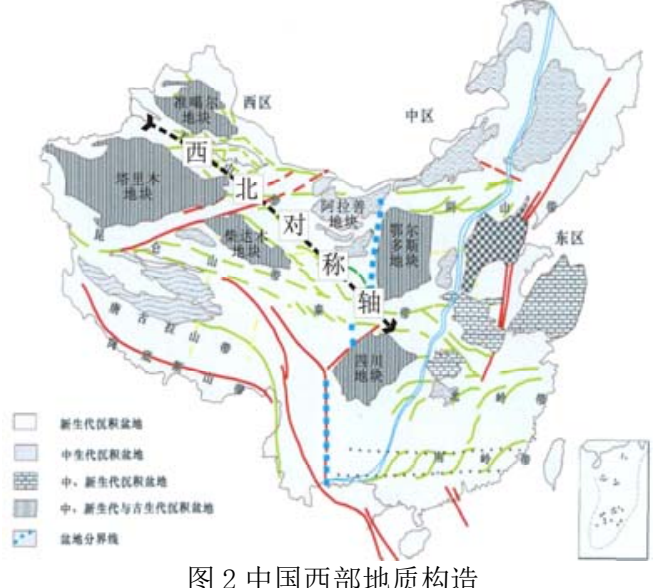

图 2 中国西部地质构造

间、华南块体与秦岭山地之间的缓冲带。此对称轴 从地形结构上来看, 位于天山山地一祁连山山地一 秦岭山地前坡。 
西北对称轴是中国最大一级的空间对称轴, 是 重大地震在两边交替活动的分界线, 这正恰似于跷 跷板的支点。西北地震带是地震活跃区, 而对称轴 则是一个相对稳定的弱作用区及地震平静带, 与郭 增建研究的时间 “静中动” 不同 ${ }^{4}$, 这是一种空间 “动中静” 的结构现象, 是一个少震的 “空心廊 道”, 是地震波浪活动的一种表现。此处, 空间对 称轴很有可能成为中国西部窥视地震活动空间规律 的重要窗口，即“非应力窗口”。

\section{2. 空间对称轴动力学基础}

已有研究发现中国西部地质构造具有对称性特 点, 新疆拜城一青海德令哈一四川广元连线对称轴 是西部地区地块之间的过渡带 ${ }^{5}$ (图 3)。准噶尔地 块、吐哈地块、阿拉善地块、鄂尔多斯地块等位于 对称轴的东北方向, 而塔里木地块、柴达木地块、 四川地块等位于对称轴的西南方向, 这些地块均属 刚性地块。 $\mathrm{Ms} \geqslant 8$ 地震基本发生在地块边缘地带, 在中心少有发生。在对称轴上, $\mathrm{Ms} \geqslant 8$ 地震发生的 次数几乎为 0 。由此说明, 中国西部地震空间对称 轴的动力学基础, 主要是由地质构造基础决定的。

新疆拜城一青海德令哈一四川广元连线对称 轴, 位于亚洲板内地震的南北应力的碰撞带后部 位, 是剪切断裂带的缓冲线, 其主要力源来自印度 板块导致的青藏块体向北运动（图 4), 这里也是 地质构造及地震活动的相对空档; 这条线附近也是

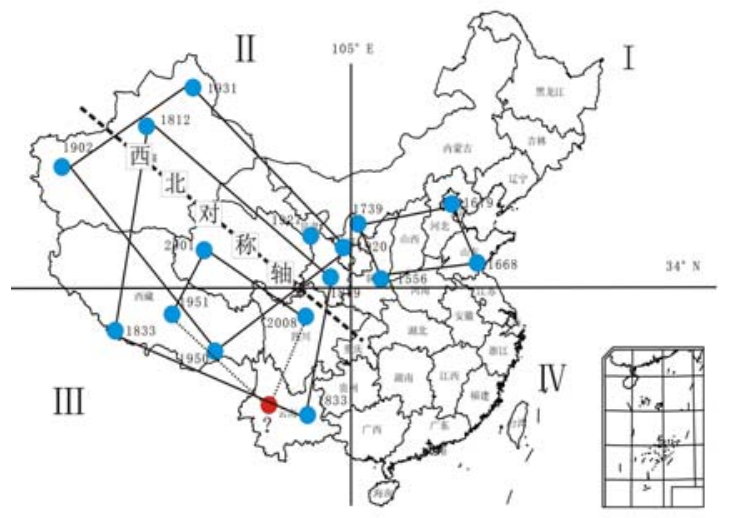

图 31500 年以来中国大陆 16 次 $M_{s} \geqslant 8$ 地震空间对称性

现今地壳运动速度比较缓慢或运动方向的转折区 域。据 GPS 站点监测 $6,7,8,9,10$, 水平运动速度场在一 定位置出现了转向, 即由西南方向转为东北方向, 而这个位置就是我们描述的西北对称轴。换言之, 该对称轴就是水平运动场的方向转折线, 是速度波 浪的波谷区。

\section{3. 云南地震空间对称轴}

\section{1. 云南地震空间对称轴特征}

云南省位于印度板块与欧亚板块中国大陆碰撞

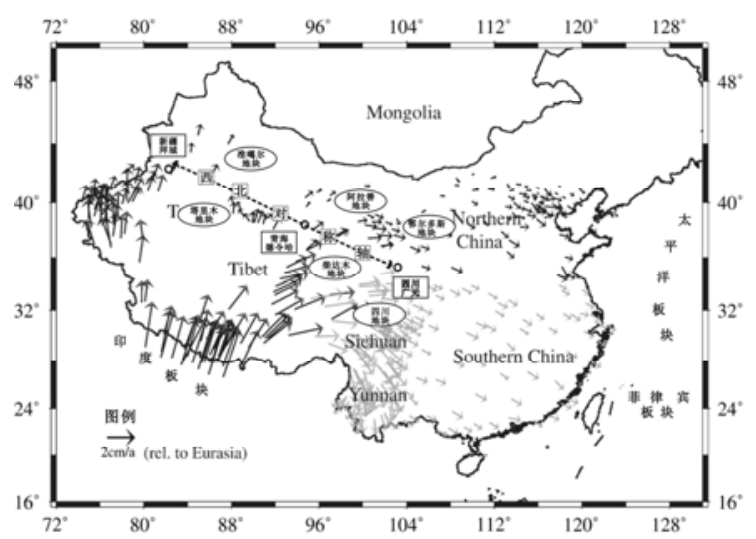

图 4 中国大陆及周边地区现今地壳运动速度场 (据文献 8 改绘)

带东缘, 地震活动频度高、震级大、分布广, 属于 板缘、板内地震混合型地区 ${ }^{11}$ 。1500 年以来, 云南 共发生 20 次 $M s \geqslant 7$ 级地震 ${ }^{12}$, 且基本发生于块体边 缘。以 $101.5^{\circ} \mathrm{E} 、 24.2^{\circ} \mathrm{N}$ 为对称轴, 4 个象限区均已 发生 5 次地震, 具有明显的对称性。总体看来, 以 三次大震的震中位置为顶点, 构成三角形, 同一三 角形的三个顶点基本出现在经向对称轴 $101.5^{\circ} \mathrm{E}$ 的 异侧(第三组除外), 而在纬向对轴 $24.2^{\circ} \mathrm{N}$ 两侧对称 性变化规律并不明显 ${ }^{13}$ 。由此可以看出, 东西震荡 性是云南地区 $M s \geqslant 7$ 级地震空间活动的主要特点,

且 $101.5^{\circ} \mathrm{E}$ 对称轴两侧地震活动相对均衡。

\section{2. 空间对称轴动力学基础}

云南地区地质结构复杂, 是不同大地构造交汇 地带, 其中可分出东南地洼区的滇桂地洼系、南北 地洼区川滇地洼系、巴颜喀拉地洼区、滇西地洼 区、腾冲地洼区。据研究, 云南地区平均热流值 高, 说明该地区地壳活动频繁, 且强度大于全国平 均值 ${ }^{14}$ 。从 $500 \mathrm{~m}$ 深地温图和 $1000 \mathrm{~m}$ 深地温图上来 看, 以 $101.5^{\circ} \mathrm{E}$ 为轴, 发现其东西两侧等温线分布 均具有东疏西密的特点。笔者推测此处存在地质构 造的分界线。

从中国西南地区现代水平活动图来看（图 5), 在 $101.5^{\circ} \mathrm{E}$ 附近存在南北向的动力来源, 中部 块体自北向南运动, 造成了块体间的错动, 从而引 发了大震。为了满足最小作用量原理, 云南 $\mathrm{Ms} \geqslant 7$ 地震呈东西震荡性分布, 两侧的地震活动发生也较 均匀。 


\section{4. 台湾地震空间对称轴}

\section{1. 台湾地震空间对称轴特征}

利用 1900 年来, 台湾及近海 $\mathrm{Ms} \geqslant 7.2$ 地震数据 （数据来源于中国强震目录）, 探讨台湾地震 $M s \geqslant 7.2$ 地震的空间对称性分布。对 17 次 $M_{s} \geqslant 7.2$ 地震进行空间标注, 共顺次形成 4 个四边形结构, 5

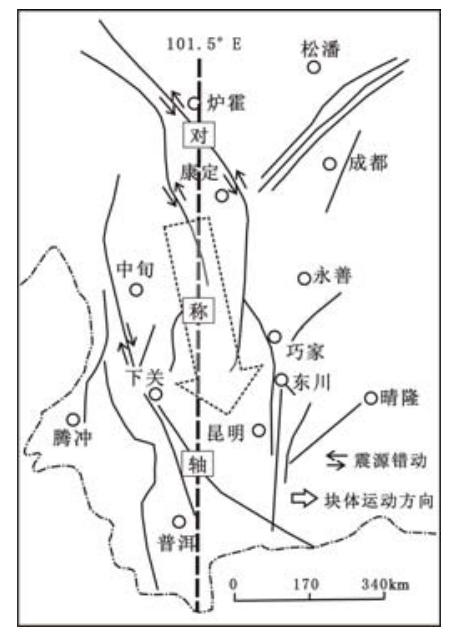

图 5 中国西南地区现代水平活动图 (据文献 15 改绘) 个三边形结构, 归纳震源点的迁移规律, 发现五个 三边形均是一个顶点在南部, 其他两点在北部, 四 个四边形的第二点均跨轴发生 ${ }^{16}$ 。

\section{2. 空间对称轴动力学基础}

台湾地震空间对称轴的动力学基础, 笔者认为
亦与台湾地区的构造分布有密切的联系。台湾地区 的构造分为北部的琉球弧沟系统和南部的吕宋弧沟 系统。图 7 为对称分布的北部的 B-D 区和南部的 AC 区, 从图中可以看到区域内的地震空间分布有较 好的对称性。特别是深源地震都分布在两个相反方 向的俯冲带前缘的冲绳海槽和吕宋海槽, 而 D、A 区域分别为琉球海沟、马尼拉海沟后的弧后盆地, 这两个区域内的地震基本都为浅源地震 ${ }^{17}$ 。

\section{5. 本研究关注核心问题}

1）强震灾害趋势判断、强震预测、强震预报的 区别是什么?

强震灾害趋势判断、强震预测、强震预报是三 个不同的概念。

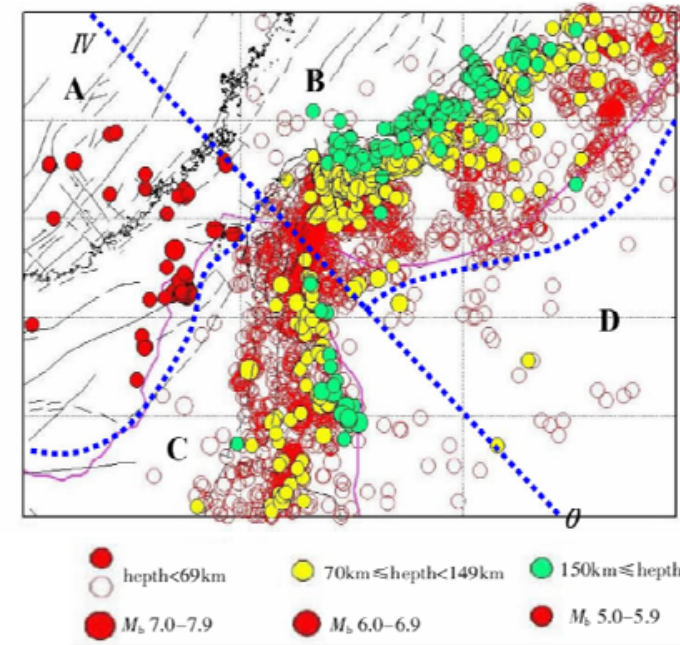

图 7 台湾地震空间对称轴分布图 (见参考文献 17)
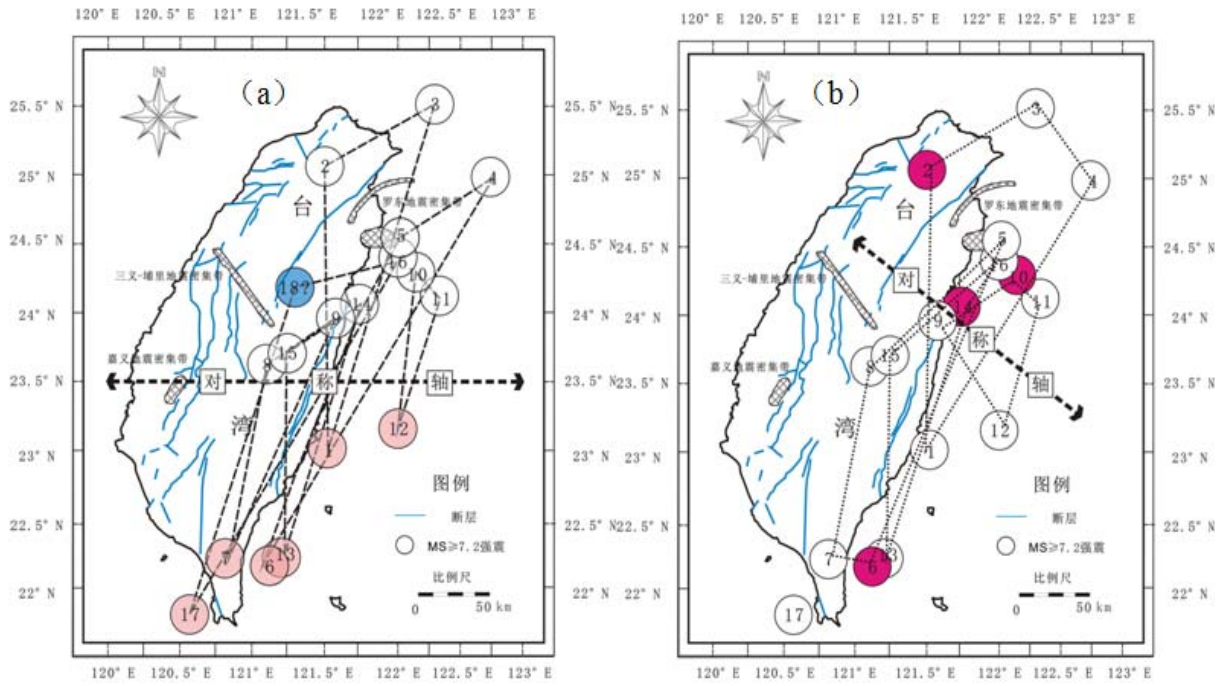

图 6 台湾 $M s \geqslant 7.2$ 地震三边形（a）、四边形（b）空间分布图（见参考文献 16） 
地震预报按照时间尺度可以分为 5 个阶段：第 一、长期预报, 指几年至几十年或更长时间内的地 震危险性及其影响的预报; 第二、中期预报, 指几 个月至几年内将要发生的破坏性地震的预报; 第 三、短期预报, 指几个天至几月内将要发生的破坏 性地震的预报; 第四、临震预报, 指几天内将要发 生的破坏性地震的预报或预警; 第五、震后震区余 震及趋势预报。

一般而言, 预报时间越长, 其预报空间范围越 大。上述预报, 特别是短期预报和临震预报, 只有 国家指定的专门机构发布, 是政府行为。无论是专 业还是业余, 个人或是集体, 对于上述地震预报内 容所持的分析结果和判定意见, 只能向有关上级主 管部门上报, 不能自行向公众发布或散布。强震预 测是有关人员为这种地震预报提供的参考资料和意 见, 《中华人民共和过防震减灾法》对地震预测和 地震预报两者概念做出了明确界定 ${ }^{18}$ 。

强震趋势判断, 是学者们研究的内容, 一般讨 论中长期地震趋势时, 即在 1 2 年或 10 年左右的宏 观地震可能性, 其地震三要素(时间、地点、震级)一 般不会十分精确, 重在给人们以预警信号。基于上 述考虑, 我们的研究定位为强震趋势判断, 而非强 震预测、更不是强震预报。

2）对称性与部分强震灾害时空结构及机理分 析, 是否可以上升到数学阶段, 构建数学模型或概 念模型, 用数学语言定量描述其逻辑关系, 将研究 由定性向定量进一步深化?

在科学研究中, 数学语言是定量化研究的最好 体现, 也是其研究成熟度的最有力说明。数学模型 是对研究内容高度抽象的概括, 是每个研究孜孜不 倦的追求, 我们研究也不例外。从目前研究现状 看, 数学模型的建立还是存在很大的困难的, 这个 定位应该是清楚的, 但是概念模型突破很有可能, 如构建对称性的概念模型, 强震灾害机理的概念模 型等, 这些都是下一步研究工作的重点和难点, 需 要进一步的努力和探索。

3) 文中列举了大量的重大自然灾害趋势判断案 例，如果存在错报、漏报，如何看待?

我们对部分重大自然灾害未来趋势做出判断, 其存在错报、漏报是可能的, 或许说是不可避免 的。我们必须坚持 “实事求是” 的态度, 是什么就 是什么, 不必刻意地追求准确, 而有意的将漏报、 错报隐去。应坚信一点, 失败不可怕, 要勇于借鉴 失败的经验。在正确的方向上, 失败是一次又一次 成功的开始。在现阶段, 对称性与自然灾害的研 究, 尚处于初步阶段, 这个定位应该是明确的。任 何理论和研究发展都需要一个过程, 这个过程有长 有短, 但都需坚持不解, 只有坚持才能有突破。

\section{4) 以对称性为切入点, 未来强震灾害发生能否 进行预测?}

国际公认, 有科学和应用意义的地震预测必须 较准确地事先估计强震震中位置、发震时间和震级 三个参数 ${ }^{19]}$ 。曾有科学工作者提出以下判断标准:

a）对地震预测依据的可观测量有定量描述;

b）对未来地震的时间、地点和震级给出定量描 述, 包括误差范围;

c）有事先预测的详细文字记录;

d）过去曾做过详细预测机理，包括成功的和失 败的（虚报、漏报）。

至今人类还不能准确预测强震发生趋势, 地震 预测研究成为全球最复杂和最具有挑战向的重大科 学和社会问题之一。

以对称性为切入点, 探讨强震灾害发生时空结 构, 借助时间对称性方法确定重大自然灾害发生时 间趋势，通过对空间对称性及时间有序性的探索， 进一步逼近灾害发生的可能区域, 是本文研究的核 心思路。我们也需要清晰的认识到, 本研究的弊端 和局限性。唯象向唯理发展是一种必然趋势, 地震 机理不明是制约地震预测的最大瓶颈。如果没有清 晰地物理机理做基础, 准确预测还是存在很大难度 的。在现有技术手段和对地震规律认识下, 对强震 未来发生趋势准确预测是难以做到的。但是, 不排 除未来关键技术的突破, 地震资料积累的丰富, 准 确预测强震的可能。我们的观点是：应该有信心， 坚信 “强震应可预测”, 只是现阶段还需要不断的 探索和积累, 在唯象基础上寻求唯理机理是一项有 意义的探索。

\section{6. 结论}

本研究以对称性为切入点, 通过大量事实积 累, 探索地理学与对称性的关系, 揭示重大自然灾 害时空对称结构内在规律, 形成公理化结论, 在唯 象基础上寻求唯理机理, 为地球科学认识对称性规 律提供一种新的思路。得到初步结论如下:

1）地震在部分地区有空间对称性现象。中国西 北地区地震存在西北一东南向空间对称轴、云南地 区地震存在南北向空间对称轴、台湾地区地震存在 西北一东南向空间对称轴。

2) 对称有一定的动力学基础。中国部分地区地 震具有空间对称性, 其存在动力学基础, 其内在物 理机理还有待进一步探索, 空间对称性应是动力学 基础和内在物理机理的外在表现, 三者应该是有机 统一的。

3）强震的对称性可以作为地震趋势判断的依 据, 为后续研究提供了启示, 但仍有待完善。笔者 认为强震的对称性是时间对称性和空间对称性的有 
机统一, 在某些情况下, 其空间对称性会在时间对 称性中得以体现, 如何有效的抓住表象对称, 进而 深入研究内部更深层次的对称机理, 是一个值得探 索的问题。

\section{致谢}

本研究得到了国家自然科学基金项目 “部分重大自 然灾害的时空对称性：结构、机理与适应对策” (41171090)的资助。.

\section{参考文献}

1. Li Shuangshuang, Yan Junping, Liu lishan, et al. Spatiotemporal symmetry and tendency judgment of the MS $\geqslant 7.8$ strong earthquake in Indonesia. Journal of Natural Disaster, (01) (2013) : 190-197.

李双双, 延军平, 刘栎杉, 等.印度尼西亚 $\mathrm{Ms} \geqslant 7.8$ 强震 时空对称特征及其趋势判断. 自然灾害学报, (01) (2013): 190-197.

2. Huang Chongfu. Natural disaster risk assessment-theory and practice. (Science Press, Beijing, 2005, 3-4). 黄崇福.自然灾害风险评价——理论与实践, 北京:科学 出版社, 2005, 3-4.

3. Yan Junping, Yan Na.Probability and verification of constructing an earthquake predicting system. Journal of Shaanxi Normal University (Philosophy and Social Sciences Edition), 37(5) 2008: 19-23.

延军平, 间娜. 关于地震预测体系构建的可能性及验证. 陕西师范大学学报(哲学社会科学版), 37(5) (2008): 1923.

4. Guo Anning, Guo Zengjian. Forecast review of WenChuan earthquake. (Xian Map Publishing Press, Xian, 2009, 148-159)

郭安宁, 郭增建. 5.12 汶川地震预报回顾, 西安: 西安地 图出版社, 2009, 148-159.

5. Liang Haihua. Occurrence conditions for great earthquakes. Seismology And Geology, 32(2) (2010): 295-301.

梁海华. 大地震发生的条件. 地震地质. 32(2) (2010): 295-301.

6. Wang Qi, Zhang Peizhen, Ma Zongjin. GPS database and velocity field of contemporary tectonic deformation in continental China. Earth Science Frontiers (China University of Geosciences, Beijing), 9(2) (2002): 416-428. 王琪, 张培震, 马宗晋. 中国大陆现今构造变形 GPS 观测数据与速度场, 地学前缘, 9(2) (2002): 416-428.

7. Wang qi, Zhang Peizhen, Niu Zhijun, et al. Crustal movement and tectonic deformation in recent Chinese mainland. Science in China (Series D), 31(7) (2001): 529-536.

王琪, 张培震等, 中国大陆现今地壳运动和构造变形. 中国科学( D 辑), 31(7) (2001): 529-536.

8. Qi Wang, Peizhen Zhang et al. Present-day crustal deformation in China constrained by Global Positioning
System (GPS) measurements. Science, (294) (2001): 574577.

9. Jian Wang, Zheng-Ren Ye, Jian-Kun He. Threedimensional mechanical modeling of large scale crustal deformation in China constrained by the GPS velocity field. Tectonophysics, (446) (2008): 51-60.

10. Ma Zongjin, Chen Xinlian, Ye Shuhua, et al. GPS research of crustal movement in recent Chinese Mainland. Chinese Science Bulletin, 46(13) (2001): 11181120.

马宗晋，陈金连，叶叔华, 等. 中国大陆现今地壳运动 的 GPS 研究. 科学通报, 46(13) (2001): 1118-1120.

11. Huang Fugang. A study on seismic activity in Yunnan area, $P h D$ thesis, Anhui: University of Science and Technology of China, (2009).

皇甫岗. 云南地震活动性研究. 中国科学技术大学博 士学位论文(2009).

12. Qin Jiazheng, Qian Xiaodong, Liu Lifang. Study on interval-time characteristics of strong earthquake activities in Yunnan region. Journal of Seismological Research, 32(3) (2009): 221-227.

秦嘉政, 钱晓东, 刘丽芳. 云南强震活动间隔时间特征 研究. 地震研究, 32(3) (2009): 221-227.

13. Yan Junping, Bai Jing, Su Kunhui, et al. Research on symmetry and tendency of several major natural disasters. Geographical Research, 30(7) (2011): 11591168.

延军平, 白晶, 苏坤慧等. 对称性与部分重大自然灾害 趋势研究. 地理研究. 30(7) (2011): 1159-1168.

14. Xu Qing, Wang Jian, Wang Jichang, et al. Terrestrial heat flow and its tectonic significance in Yunnan, China. Geotectonica Et Metallogenia, 16(3) (1992): 285-299. 徐清, 云南大地热流及其大地构造的意义. 大地构造与 成矿学. 16(3) (1992): 285-299.

15. Guo Anning, Chen Jiachao. Problem on strong earthquake in China. (Earthquake Press, Beijing, 2002). 郭安宁, 陈家超. 中国大震问题. 北京: 地震出版社,2002.

16. Yan Junping, Li Shuangshuang, Liu Xinyan, et al. The trend judgment of earthquake in Taiwan based on symmetry theory and its physical basis. Journal of Catastrophology, 28(1) (2013): 11-14. 延军平, 李双双, 刘新颜, 等. 基于的中国台湾地震趋势 判断及物理基础, 灾害学, 28(1) (2013): 11-14

17. Chen Daqing, Yang Maling. The Symmetrical Partition of Taiwan Earthquake and its Relation to ModerateStrong Earthquakes in Adjacent Area of Guangdong and Fujian Provinces. South China Journal of Seismology, 30(sup.) (2010): 69-75. 陈大庆, 杨马陵. 台湾地震对称分区及与粤闽交界中强 地震的对应关系. 华南地震, 30(增刊) (2010): 69-75.

18. Ma Zongjin. Question of earthquake knowledge. (Science Press, Beijing, 2008).

马宗晋. 地震知识问答. 北京: 科学出版社,2008. 